\title{
Einleitung
}

\section{Komplex Familie}

mein famili

mein schwester strickt am grünen strumpf/so heiß und groß so dick und weich/so seltsam übers knie gebeugt / mein schwester mit dem roten numpf

mein oma liebe oma so / so faltig pergament so dünn / so vogel hals so fistel stimm / so mürrisch mittags abends froh

mein famili im zimmer lung / wo um den schwarzen tisch und rund / mit topf und fisch und zwiebelbrüh / mit hand mit mund mit großem hung

mein vater mit der nickelbrill / mein mutter auf dem küchenstuhl / mein starker bruder mit dem bart / mein kleine schwester blaß und still

mein famili mein ganze fam / ili mein ganze zwei drei und / mein vier und fünf und zwei und ein / mein familie wie wundersam

wie wundersam wie wir am tisch / am runden tisch von rundem holz / wie faust und gabel hier und hier/faust gabel hier mund da und fisch

mund da und fisch und fisch und kloß / am runden tisch der vater spricht / und ißt den kloj und $i \beta t$ den fisch / vom tisch und spricht von zwiebelsoß

und zwiebelsoß rinnt ab vom mund / wischt ab ach wischt und wischt und spricht / die schwester hörts an ihrem strumpf/die mutter hörts der bruder und

großvater dort auf dem abort / der hund der hund bunt hinterm schirm / die laus in seinem pelz und ich / ich hörs und hab im ohr die wort

wie altes brot die wort wie brot / wie schwarzes brot die mutter nimmts / vom küchenbord und lächelt wild / denn trocken brot macht wangen rot

wie glotzt aus seinem leib das brot / wie rollt es rollt es durch die tür/die base sitzt vor dem klavier/und spielt ohn brot ist große not

die schürz der mutter weht herum / die uhr platzt an der wand und da /grinst unterm bottich grinst die schab / der vater spricht seht euch nicht um (ror wolf, 1968)

In dieser Moritat hat ror wolf 1968 etwas anklingen lassen, was sich mit dem „Komplex Familie“ assoziieren ließe. Mögen manche Bilder in „mein famili“ - psychohistorisch betrachtet - auf das letzte und sogar vorletzte Jahrhundert zurückverweisen, es bleibt die Atmosphäre des Heimelig-Unheimlichen einer nährenden Instanz, die für die Familienmitglieder zu einem Zentrum von Attraktion und Repulsion wird, es bleibt der an die Familie gebundene Erinnerungsspeicher, der allerdings oft genug nur das hohle Echo von „Lebensweisheiten“ enthält. Diese Instanz lässt sich nicht hintergehen, bei aller Abwehr gegen ihre repressiven und restriktiven Züge werden die Meisten sich an bestimmten Punkten der eigenen Biographie immer wieder neu damit konfrontiert sehen, was Familie (ihnen) zu bedeuten hat und Entscheidungen treffen müssen, wie sie für sich das gestalten wollen, was ,Fa- 
milie' genannt wird. Immerhin haben sich die individuellen Gestaltungsspielräume geweitet, seit Sigmund Freud über den „Familienroman der Neurotiker“ (1908) und damit über einen Vorgang schrieb, der heute als normaler Bestandteil von Entwicklungspsychologie und keineswegs mehr zwangsläufig als neurosenbildend gilt. Doch ist der Komplex Familie deshalb nicht weniger emotional besetzt, wie sich in den letzten Jahren unter anderem an öffentlichen Debatten über die famiale Arbeitsteilung gezeigt hat, in denen man sich auf eine Biologie der Familie berief.

In einer kritischen Auseinandersetzung mit dem Stellenwert von Familiensoziologie als theoretischem Terrain schrieb Günter Burkhart 2006, möglicherweise habe „Familie - wie Geschlecht oder Lebenslauf - mehr den Charakter einer omnirelevanten Perspektive als den eines sachlich klar abgrenzbaren Gegenstandsbereichs“ (Burkhart 2006, 175). Ja es gebe sogar Zweifel, ob die Bezeichnung „Familiensoziologie" nach dem Bedeutungsverlust der klassischen Familie noch zutreffend sei. „Doch über die Alternative gibt es keine Einigkeit, und man kann dies auch als Symptom einer theoretischen Unsicherheit betrachten. Ist Familie eine Institution mit bestimmten Normen und Funktionen? Ist sie ein System (oder Teil eines anderen Systems oder besteht sie aus Teilsystemen)? Ist sie eine von mehreren Lebensformen oder sozialen Beziehungstypen, eine neben anderen oder doch noch eine hervorgehobene Form?"“ (Burkart 2006, 177). Sobald man also dem Komplex Familie begrifflich zu Leibe rücken will, stößt man auf neue Fragen.

Was könnte Familie aus feministischer Perspektive heute sein? Individuell wird sie unabhängig von sexueller Orientierung häufig als Ort der Privatheit, der Liebe und der Verbundenheit gedacht. Gerade in der heutigen Zeit, die auf ein Individuum setzt, das seines Glückes Schmied ist, erscheint die Familie (wieder) als ein Fluchtpunkt, als ein Ort der Gemeinsamkeit mit dem Versprechen auf Liebe und Fürsorge, ein Ort, an dem andere Regeln herrschen als in der Arbeitswelt. Ausgeblendet bleiben dabei oft die anderen Seiten von Familie, wie Machtungleichgewichte und Gewaltverhältnisse.

Die Vorstellung der bürgerlichen Gesellschaft von einer strikten Trennung zwischen Erwerbsarbeit und Familic war schon immer eine Fiktion, wie die feministische Forschung der 1970er und 1980er Jahre zeigen konnte. Mittlerweile sind die Sphären von Erwerbsarbeit und Familie noch weniger gegeneinander abzudichten, hat der gesellschaftliche und ökonomische Wandel die Grenzen zwischen Arbeit und Leben, Privatem und Öffentlichem noch durchlässiger werden lassen. Die zunehmenden Anforderungen der Arbeitswelt an Mobilität und Flexibilität männlicher wie weiblicher Erwerbstätiger haben sich auch im Familienleben niedergeschlagen. Zeitliche und räumliche Flexibilisierungsprozesse in der Erwerbsarbeit haben dazu gefuihrt, dass Familienleben heute häufig in den knappen Zeitlücken der überbordenden Erwerbsarbeit gleichsam auf Knopfdruck stattfinden muss. Angesichts der Tatsache, dass viele Familien gar nicht am selben Ort leben, ist dazu oft auch die Überwindung beträchtlicher räumlicher Distanzen erforderlich. Von den Grenzverschiebungen zwischen Arbeit und Familie blieb die 
,Institution Familie nicht ausgenommen. Familie kann nicht als selbstverständlich vorausgesetzt werden, sie ist kein statisches Gefüge oder hierarchisch strukturiertes Generationennetzwerk, sondern etwas, das hergestellt werden muss und in der alltäglichen Lebensführung in verschiedenen Formen gelebt wird. Diese Idee von Familie als „Herstellungsleistung“ hat seit dem siebten Familienbericht der Bundesregierung (2006) an Boden gewonnen. Zwar sei der Begriff vorerst mehr Programm als ausgearbeitetes Konzept, aber er transportiere sowohl eine sozialhistorische als auch eine konzeptuelle Botschaft, nämlich die, dass sich Familie aus einer quasi naturgegebenen Ressource in eine voraussetzungsvolle Aktivität von Männern, Frauen, Kindern, Jugendlichen und alten Menschen verwandelt habe, die in ihr leben wollen. „Familie als Herstellungsleistung fokussiert zum einen auf die Prozesse, in denen im alläglichen und biographischen Handeln als gemeinschaftliches Ganzes permanent neu hergestellt wird (Doing Family), zum anderen auf die konkreten Praktiken und Gestaltungsleistungen der Familienmitglieder, um Familie im Alltag lebbar zu machen. Der Tätigkeits- oder Arbeitscharakter von Familie, der eigene Ressourcen bindet, wird damit - weit über die feministische Hausarbeitsdebatte hinausgehend - sichtbar, ohne jedoch die emotionale Bedeutung von Familie zu schmälern. Konzeptuell bedeutet die Rede von Familie als Herstellungsleistung eine stärkere Fokussierung des Handlungsparadigmas gegenüber dem institutionellen Paradigma." (Schier/Jurczyk 2007, 10)

Diese Vorstellung von Familie, die sich auf empirische Forschungen stützt, ist nicht auf die so genannte Kernfamilie begrenzt, sie vermag das ganze Spektrum sogenannter neuer Lebensformen, von Wahlfamilien in Wohngemeinschaften, ,Patchworkfamilien', Einelternfamilien, eingetragenen Lebenspartnerschaften mit oder ohne Kinder etc. zu integrieren. Auch die theoretische Familiensoziologie muss inzwischen anerkennen, dass von einem fortbestehenden unveränderten Familienleitbild und insofern auch von einem Kollektivsingular Familie nicht mehr die Rede sein kann und zwar hauptsächlich deshalb, weil Partnerschaft und Elternschaft sich in den letzten Jahrzehnten zunehmend auseinanderentwickelt haben (vgl. Tyrell 2006).

Das Heft Komplex Familie will diesen Wandel in der Praxis der Familie ebenso thematisieren wie die wissenschaftliche Reflexion über diesen Prozess. Ute Gerhard nimmt in ihrem Beitrag die neue Pluralität der privaten Lebensformen in den Blick und diskutiert Familienpolitik und Familienrecht im Kontext von Freiheit und Gerechtigkeit für die Einzelnen. Die widersprüchlichen Botschaften und paradoxen Folgen der westdeutschen Familienpolitik verdeutlicht sie am Beispiel des Umgangs mit der Müttererwerbstätigkeit, dem neuen Unterhaltsrecht und der Rechtsformigkeit alternativer Lebensformen. Gegen das Defizit an fürsorglichen Tätigkeiten fordert sie eine Care-Verpflichtung aller Menschen. Gerhards feministisches Argument in Bezug auf die Familie lautet, dass Liebe und Gerechtigkeit nicht in Opposition stehen (dürfen), sondern im Gegenteil Liebe eine Praxis von gleichen Rechten voraussetzt. 
Nicht nur Familie und Familienrecht haben sich verändert, auch die bundesrepublikanische Familienpolitik hat sich angesichts des seit einigen Jahren als dramatisch wahrgenommenen demographischen Wandels auf ,neue Aufgaben besonnen. Obwohl zum Teil schon vorher eingeleitet, sind besonders unter der Regie der CDU-Familienministerin von der Leyen Veränderungen auf den Weg gebracht worden. Wie ist diese neuere deutsche Familienpolitik einzuschätzen und in welchem Verhältnis steht sie zur Gleichstellungspolitik? Mechthild Veil geht diesen Fragen nach. Sie betont, dass es zu kurz greifen würde, die Familienpolitik nur als Produkt eines neuen Konservatismus zu interpretieren oder als neoliberale Position, die sich von früheren Forderungen feministischer Bewegungen verabschiedet oder sie in verkürzter Form vereinnahmt habe. Sie analysiert die seit der Jahrtausendwende eingeleitete familienpolitische Wende als Reaktion auf einen ,Gleichstellungsdruck', als nachholende Modernisierung im Sinne einer Aufholbewegung gegenüber anderen europäischen Ländern. Darüber hinaus möchte sie zur Diskussion stellen, inwieweit eine von ökonomischen Interessen dominierte Familienpolitik, bei der es um die Rekrutierung und Bindung qualifizierter weiblicher Arbeitskräfte geht, nicht auch gleichstellungspolitische Relevanz haben kann. - Festgestellt werden kann, dass die ,neue' Familienpolitik an der demographischen Entwicklung in Deutschland bislang wenig geändert hat, wenn auch nach Einfuhrung des Elterngeldes 2007 ein geringfuigiger Geburtenanstieg zu verzeichnen war.

Patrick Ehnis und Sabine Beckmann befassen sich mit einem Kernstück der Familienpolitik unter Ursula von der Leyen, dem Elterngeld. Sie bringen in ihrem im Diskussionsteil aufgenommenen Beitrag die Perspektive von Vätern ein. Aus der Sicht von ,aktiven'Vätern, die Elternzeit in Anspruch nehmen, beurteilen und kritisieren sie das Bundeselterngeld und -zeitgesetz. Das als Lohnersatzleistung konzipierte Elterngeld sei zu kritisieren, weil die Betreuungsarbeit nicht nach ihrer Qualität als gesellschaftlich notwendige Arbeit, sondern in Ableitung von der vorher erbrachten Erwerbsarbeit bewertet wird. Damit werde nicht nur die über Erwerbseinkommen vermittelte soziale Unglcichheit fortgeschrieben - wie sich am deutlichsten daran zeigt, dass nach den neuesten Sparbeschlüssen der Bundesregierung das Elterngeld für Hartz-IV-Empfänger gestrichen wird -, sondern auch eine Arbeitsteilung zwischen den Geschlechtern, in der die Berufsrolle des Mannes immer noch als zentraler Teil seiner Familienrolle gilt. Das hat zur Konsequenz, dass Elternschaft für Frauen und Männer nach wie vor Unterschiedliches bedeutet: „Für Frauen besteht sie in der alltäglichen Praxis der Betreuung und Erziehung der Kinder, für Männer ist sie primär ein sozialer Status, der durch die außerfamilialen beruflichen Aktivitäten gewonnen wird. [...] Vaterschaft manifestiert sich nicht in einem Engagement in der Familie, sondern für die Familie: in der Erfüllung der Ernährerrolle.“ (Meuser 2008) Noch immer spielt dieses Modell für männliche Selbstbeschreibungen vor allem bei Führungskräften eine wesentliche Rolle. 
Pamela Heß diskutiert in ihrem Beitrag, dass trotz der gravierenden sozialen, politischen und ökonomischen Transformationen nach 1989 die unterschiedlichen Vorstellungen über Müttererwerbstätigkeit und familiale Arbeitsteilung in Ost- und Westdeutschland erhalten geblieben sind und die Arbeitsteilung in der Familie nach wie vor unterschiedlich gelebt wird. Ihre sekundäranalytische Auswertung des Familiensurveys zeigt, dass sich die praktizierte Arbeitsteilung in den alten Bundesländern stärker gewandelt hat als in den neuen Bundesländern, wo die Mütter trotz des Strukturwandels an ihrer starken Erwerbsorientierung festhielten. Diskutiert werden diese Befunde mit Blick auf (un-)gleiche Geschlechterkulturen in West- und Ostdeutschland.

Die Widersprüche und Ambivalenzen beim Thema Ehe und Familie werden besonders deutlich, wenn es um die Gleichstellung von lesbischen und schwulen Partnerschaften im Eherecht geht. In den Auseinandersetzungen um die Eingetragene Lebenspartnerschaft in Deutschland wurde auch von Linken kritisch gefragt, warum gerade Lesben und Schwule eine Gleichstellung in Bezug auf die konservative Institution Ehe für sich reklamieren, statt diese Institution generell in Frage zu stellen. Anders verliefen dagegen die Auseinandersetzungen um die gesetzliche Regelung in Frankreich. Dort existiert seit 1999 die rechtliche Anerkennung eines Pacte Civil de solidarité (PACS), ein Solidaritätsvertrag, der allen Paaren offen steht und nicht an die sexuelle Orientierung der PartnerInnen gebunden ist. Genutzt wird der PACS wie Julia C. Sucks in ihrem Beitrag zeigt, interessanterweise überwiegend von heterosexuellen Paaren. Sucks vergleicht das französische und das deutsche Modell unter dem Aspekt von Antidiskrimierungspolitik und diskutiert, ob und inwieweit diese Modelle zu einer Veränderung der Institution der Ehe beitragen können. - Unter dem Aspekt der europäischen Rechtsangleichung ist es in diesem Zusammenhang von mehr als anedoktischem Wert, dass ein heterosexuelles Paar aus Linz eine homosexuelle Lebensgemeinschaft begründen will. In Österreich gibt es seit dem 1.1.2010 die Eingetragene Lebenspartnerschaft nur für Personen gleichen Geschlechts. Sollte sich das Paar mit seiner Klage durchsetzen, müsste der Gesetzgeber eine der Ehe gleichgestellte Lebenspartnerschaft einführen, in der die jetzt bei der Ehe gegenüber der eingetragenen Partnerschaft noch bestehenden Nachteile - Scheidung nur nach dem Schuldprinzip, höherer Unterhalt - ebenso wie die den homosexuellen Lebenspartnerschaften auferlegten Einschränkungen - keine Doppelnamen, keine Adoption von Kindern, keine standesamtliche Trauung - entfallen würden (vgl. Rakete-Dombeck 2010).

Selbst unter den Vorzeichen der nun von einer breiteren Öffentlichkeit begrüßten sogenannten Vereinbarkeit von Familie und Erwerbsarbeit, bleibt Mutterschaft ein Thema normativer Diskurse. Es gibt in Deutschland eine Nationale Stillkommission (NSK), die am Bundesinstitut fuir Risikobewertung angesiedelt ist. Diese Kommission hat zum Ziel, das Stillen wieder populärer zu machen. Wie schon bei der Begründung der Geschlechtscharaktere im 18. Jahrhundert (vgl. Hausen 1976) wird auch hier mit der ,Natur" argumentiert. Stillen soll wieder 
als das angesehen werden, was es sei - „einer der natürlichsten Vorgänge überhaupt". Stillen sei sexy und elegant, so lautet die Überschrift eines Berichtes über ein Symposium der Stillkommission anlässlich ihres 10jährigen Bestehens (vgl. Baumgarten 2004). Dass und wie das Stillen normativ konstruiert wird, zeigen die Autorinnen Marion Ott und Rhea Seehaus. Sie untersuchen diskurs- und praxisanalytisch, wie das Stillen, das in Ratgebern und programmatischen Schriften als Anforderung formuliert wird, in Routine-Kindervorsorgeuntersuchungen in Arztpraxen im Gespräch mit den Müttern thematisiert wird und welche „eigenwilligen Dynamiken“ dabei entstehen.

Im Diskussionsteil widmet sich Christine Ivanov einem anderen Aspekt von (normierter) Mutterschaft. In ihrem Literaturbericht über transnationale Mutterschaft betont sie, wie eng dieses Thema mit Theorien und Vorstellungen zu Mutterschaft verbunden ist und wie diametral das Phänomen transnationaler Mutterschaft diesen entgegensteht. Sie gibt einen ausfuihrlichen Überblick zum aktuellen Forschungsstand über transnationale Mutterschaft und diskutiert Studien, die überwiegend im Kontext von Untersuchungen zu Arbeitsmigration angesiedelt sind.

Wenn einerseits beklagt wird, Modelle alternativer Männlichkeit und neuer Väterlichkeit fänden zu wenig gesellschaftliche und politische Anerkennung, so gibt es doch andererseits gar nicht wenige Diskurse, die sich mit einer so genannten Krise der Männlichkeit beschäftigen. Anne Fleig hat sich mit narrativen Konstruktionen des (vorläufigen) Endes moderner Männlichkeit nach dem Muster des Zerfalls eines ,Hauses' in Thomas Manns Buddenbrooks in zwei neueren Familienromanen beschäftigt. Am Beispiel der Romane „Houwelandt" von John von Düffel (2004) und „Es geht uns gut" von Arno Geiger (2005) zeigt sie, dass das Haus zum „Spiegel der jeweiligen Familien- und Geschlechterbeziehungen“ wird und gleichzeitig das Muster der zu rekonstruierenden Familienerzählungen bildet. In der Darstellung einer Spanne von drei Generationen wiesen die Romane Gemeinsamkeiten auf, die als Reflex auf die Veränderungen der Nachkriegszeit zu verstehen seien. Allerdings seien die Auswege aus der Krise der Männlichkeit in diesen Romanen grundvcrschieden, was nicht nur mit den unterschiedlichen geographisch politischen Voraussetzungen der Autoren zu tun hat.

Der Beitrag von Heide Schlüpmann in der Rubrik „Außer der Reihe“ geht aus einer historisch-philosophischen Perspektive auf das Verhältnis zwischen Subjektphilosophie und Geschlechterdifferenz ein und versucht eine feministische Subjekttheorie mit ihren Emanzipationsversprechen jenseits essentialistischer und transzendentaler Subjekttheorien wieder freizulegen. Für die Autorin hat ihr Wechsel von der Philosophie zum Kino wegen seines besonderen Zugangs zur Wahrnehmung der Realität eine andere Aneignung der philosophischen Tradition ermöglicht.

In der Rubrik „Bilder und Zeichen“ zeigen wir Fotografien der in Berlin lebenden italienischen Künstlerin Daniela Comani. In ihrer Serie „Eine glückliche Ehe" sehen wir auf den ersten Blick Szenen aus dem Alltag eines jungen heterose- 
xuellen Paares. Die Ähnlichkeit der abgebildeten Personen führt jedoch zu leichten Irritationen. Erst genaues Hinsehen lässt erahnen, dass Comani mit Geschlecht spielt, dass es sich auf den Fotografien immer um ein und dieselbe Person handelt. Kommentiert werden die Bilder durch einen Beitrag von Hanne Loreck.

In diesem Heft wird die schon fast in Vergessenheit geratene Rubrik „Archiv“ wieder einmal mit einem Beitrag zum Leben erweckt. In dieser unregelmäßig erscheinenden Rubrik drucken wir Beiträge ab, die einst Diskussionen anregten oder auch als Grundlagentexte wichtig geworden sind. Claudia Gather schlug den kurzen und prägnanten Kommentar von Helena Z. Lopata und Barrie Thorne mit dem Titel On the Term ,Sex Roles' aus dem Jahr 1978 vor. Dieser Text wird als frühe Kritik am Begriff der Geschlechtsrolle in den USA immer wieder zitiert, im deutschsprachigen Raum ist er dagegen weniger bekannt. Der vor allem in der deutschen Familiensoziologie gelegentlich wenig reflektierte Gebrauch des Begriffs der Geschlechtsrolle, zeigt, dass das Thema trotz der mittlerweile vorliegenden gründlichen Kritik besonders seitens Vertreterinnen des Konstruktionsansatzes (z.B. Angelika Wetterer) noch nicht überholt ist.

Abgerundet wird das Heft wie immer durch Tagungsberichte und Rezensionen.

Wir möchten Christina Koch für ihre Mitarbeit bei der Produktion dieses Heftes danken.

Claudia Gather, Regine Othmer, Ulla Wischermann

\section{Literatur}

Baumgarten, Katja (2004): Tagungsbericht: Stillen - sexy und elegant. In: Deutsche Hebammenzeitschrift http://www.viktoria11.de/artikel/Still-Symposium_Berlin.html

Burkart, Günter (2006): Positionen und Perspektiven. Zum Stand der Theoriebildung in der Familiensoziologie. In: Zeitschrift für Familienforschung 2, 175-210.

Hausen, Karin (1976): Die Polarisierung der „Geschlechtscharaktere“ - Eine Spiegelung der Dissoziation von Erwerbs- und Familienleben. In:Werner Conze (Hrsg.): Sozialgeschichte der Familie in der Neuzeit Europas. Neue Forschungen. Stuttgart, 363-393.

Rakete-Dombeck, Ingeborg (2010): Aktuelle Entwicklungen im Familienrecht. Von A wie Abkommen bis $\mathrm{Z}$ wie Zuwendung. In: Neue Juristische Wochenschrift. 63. Jg.. Heft 19, 1313-1317.

Meuser, Michael (2008):Vaterschaft im Wandel. Perspektiven aus der Männlichkeitsforschung. In: Beziehungsweise ,15. September; hrsg. v.: Österreichisches Institut für Familienforschung. Universität Wien. http://www.oif.ac.at/presse/bzw/artikel.asp?Rubrik=3\&ID_Art=1\&BZWArtikel=1029.

Schier, Michaela/Jurczyk, Karin (2007): Familie als Herstellungsleistung in Zeiten der Entgrenzung. In: Aus Politik und Zeitgeschichte 34,10-16.

Tyrrel, Hartmann (2006): Familienforschung - Familiensoziologie: Einleitende Bemerkungen. In: Zeitschrift für Familienforschung 2, 1-9.

Wolf, Ror (1968): Mein Famili. Zwölf Moritaten von Raoul Tranchirer, Giessen. 